\title{
Osteosarcoma -An Osteolytic Lesion inthe Posterior Mandible -A Case Report With Review of Literature
}

\author{
AvinashTamgadge MDS (Prof \& HOD) ${ }^{1}$ Sandhya tamgadge ${ }^{1}$ MDS (Prof),Swati \\ Gotmare ${ }^{1}$ MDS (Associate professor) ,Sudhir Bhalerao ${ }^{1}$ MDS(Prof), \\ Dr D. Y. Patil Dental College \& Hospital, Sector 7, Nerul, Navi Mumbai, Maharashtra, India
}

\begin{abstract}
Osteosarcoma is a malignancy of mesenchymal cells able to produce osteoid or immature bone. Osteosarcoma of the jaw is uncommon and represents 6-8\% of all osteosarcomas. There are 3 conventional types of osteosarcoma of the jaw i.e. osteogenic, chondroblastic and fibroblastic. Fibroblastic variant scarcely discussed in dental literature as it is extremely rare in the oral cavity and exhibits diverse clinical, radiological and histopathological manifestations resulting in misdiagnosis and diagnostic delay.

This article reports a case of fibroblastic variant of osteosarcoma in the mandible of a 30 years old female with changing radiographic patterns which resulted in recurrence due to misdiagnosis.
\end{abstract}

Key Words:osteosarcoma, , fibroblastic type, osteolytic, herring bone pattern, special stains, IHC

\section{Introduction}

Osteosarcoma has been recognized for almost two centuries and is the most commonmalignancy of the skeletal system ${ }^{1}$

It is an aggressive cancerous neoplasm arising from primitive transformed cells ofmesenchymal origin that exhibit osteoblasticdifferentiation andproduce malignant osteoid . It accounts for approximately $20 \%$ of sarcomas but only 5\% occur in jaws. Osteosarcomas of jaw are uncommon and despite its histopathologic similarities with long bonesit is biologically different. They are locally aggressive and tend to recur. ${ }^{2,3}$ There are 3 traditional subdivisions of osteosarcoma i.e., osteoblastic, chondroblastic, and fibroblastic osteosarcoma. ${ }^{4}$

Fibroblastic osteosarcoma isvery rare and is characterized by the presence of spindle cells with minimal amounts of osteoid matrix. ${ }^{5}$

Osteosarcoma may arisede novoor subsequent to previous irradiation and pre-existing disease of bone. The reported case appears to have developed de novo; as no history of any pre-existing diseasecould be elicited. ${ }^{5}$

Dental professionals may be the first to detect jaw osteosarcomas in their initial stages but it often goes unnoticed, thus stressing on the need for an early diagnosis before its confirmation by histopathology $6,7,8$

We present here a unique case of fibroblasic variant of osteosarcoma arising de novo in posterior mandible in 30 year old female which is a rare entityin jaws showing diversehistopathologic and radiographic findings.

\section{Case report}

A 30 -year-old, female patient referred at our institute with a chief complaint of a swelling on the right side of the mandible that had recurred after two months of conservative excision.

According to the referral she initially noticed the swelling at the site of extraction of lower right second molar which was removed one year back. Medical as well as systemic examinations did not reveal abnormal clinical findings.

On extra oral examination,recurrent lesionwas diffuse, bony hard and roughly about $6 \times 5 \mathrm{~cm}$ in size at the angle of the mandible. The skin overlying the swelling was of normal colour but had a glossy appearance, probably due to tautness of the skin over the swelling.(Fig 1,2)

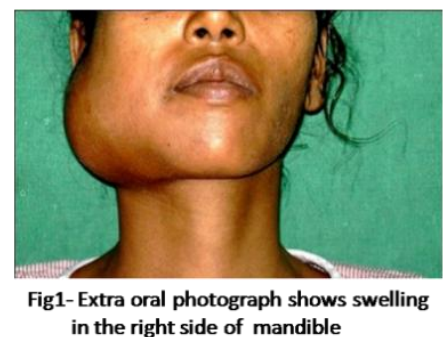

in the right side of mandible

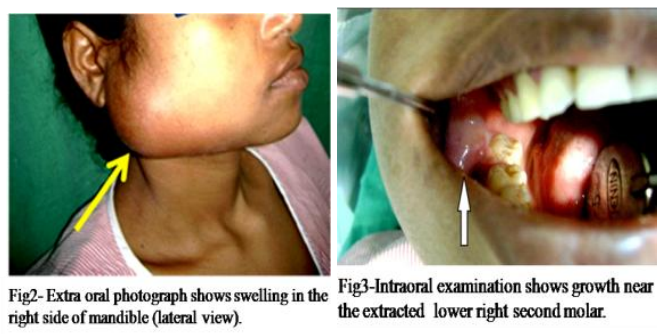


Intraorally, the swelling was evident on the mandibular buccal vestibule extending from 46 region to the ascending ramus. Bidigital palpation did not reveal any lymph node enlargement anywhere in the cervicofacial chain. (Fig.3)

The recurrent swelling was completely radiolucent(fig.4), but according to referral the firstlesion mimicked fibrous dysplasia with mixed radiolucency and radiopacity. A CT scan (fig.5) and 3D reconstruction (fig.6) of the lesion showed lytic area. However MRI could not be done due to financial limitations, as the patient belonged to a lower socio-economic group.

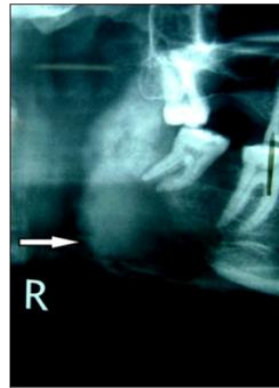

Fig 4-Lateral oblique radiograph shows radiolucent lesion.

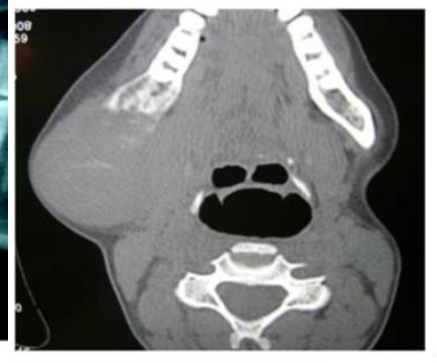

Fig5-CT SCAN of the swelling

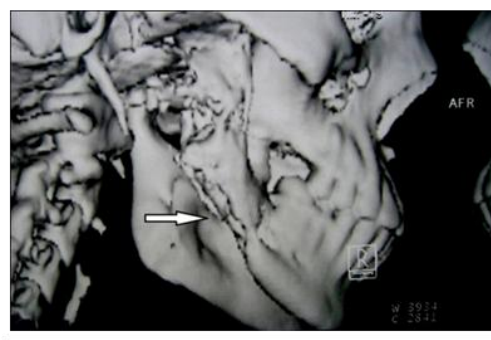

Fig6-3D reconstruction of CT scan .

Clinical findings were dissonant with the previous diagnosis of fibrous dysplasia. An immediate hemi mandibulectomy was performed (fig.7) based on the clinical features, computed tomography, to avoid encroachment of the adjacent structures .Subsequent microscopic examination revealed primarily of spindleshaped highly polymorphic cells showing "herring bone pattern", proliferating in an intertwining fascicular fashion with multinucleated giant cells. In addition, scanty hyalinised material resembling neoplastic osteoid was observed. The findings were most consistent with a fibroblastic osteosarcoma. (fig.8)
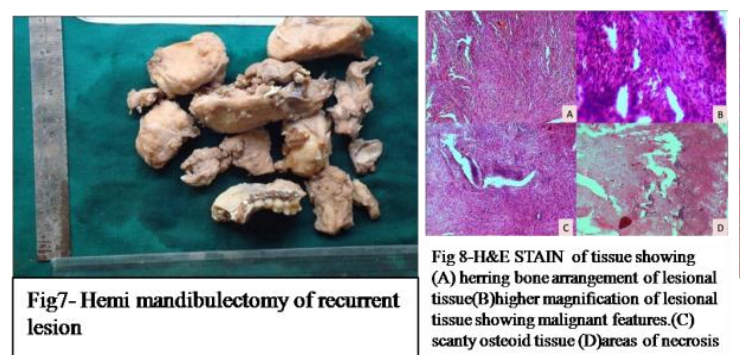

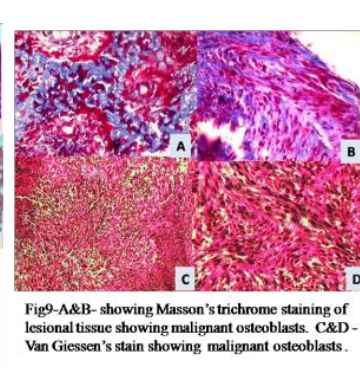

Masson's trichrome and van Giessen stains were also performed which showed prominently malignant fibroblast, osteocytes and osteoblasts (fig.9). IHC was positive for vimentin (fig10). Features confirmed the diagnosis of fibroblastic variant of osteosarcoma. Radicle resection of the tumour under general anaesthesia followed by chemotherapy was planned but the patient refused surgery. The Patient was motivated to undergo surgery, but she was reluctant and did not turn up for follow-up and she died within few months at her native place.

\section{Discussion}

The term "osteosarcoma" as opposed to "osteogenic sarcoma" is preferred by the World Health Organization (WHO).The term "sarcoma" was introduced by the English surgeon John Abernathy in 1804 and was derived from Greek roots meaning "fleshy excrescence". In 1805, the French surgeon Alexis Boyer (personal surgeon to Napoleon) first used the term "Osteosarcoma $7,9,10,11$

Although the number of craniofacial osteosarcomas is very low, the prevalence of jaw osteosarcoma is in fact 10 times greater than that of osteosarcoma in the total body skeleton, considering that jaws represent only $0.86 \%$ of total body volume. Jaw osteosarcoma have been reviewed by various authors with rarely reported fibroblastic variant in the literature ${ }^{12-20,}$ (Table 1).

They commonly present in the third or fourth decades' Males slightly out number females but the present case occurred in female ${ }^{21}$. The mandible is involved more frequently than the maxilla with posterior body, horizontal ramus and the ascending ramus being the most commonly affected site as seen in our case. 
Osteosarcoma -An Osteolytic Lesion In The Posterior Mandible -A Case Report With Review of literature...

\begin{tabular}{|c|c|c|c|}
\hline S.NO & AUTHORS & PERIOD & $\begin{array}{l}\text { TOTAL .NO OF } \\
\text { PATIENTS }\end{array}$ \\
\hline 1. & Schwatz and Apert 1963 & 1940-1961 & 66 \\
\hline 2. & Potdar 1970 & 1970 & 14 \\
\hline 3. & Roca et al 1970 & 1970 & 20 \\
\hline 4. & Caron et al 1971 & 1930- 1966 & 43 \\
\hline 5. & Russ and Jesse 1980 & 1948- 1977 & 30 \\
\hline 6. & Raymond et al 1989 & -1989 & 55 \\
\hline 7. & Colmenero et al 1990 & 1968-1988 & 15 \\
\hline 8. & Goepfert et al 1990 & 1953- 1987 & 70 \\
\hline 9. & Doval et al, 1997 & 1986- 1992 & 8 \\
\hline 10. & Oda et al. 1997 & $1981-1996$. & 17 \\
\hline 11. & Vege et al. 1991 & $1963-1981$ & 34 \\
\hline 12. & van Es et al. 1997 & 1964 to 1992 & 46 \\
\hline 13. & Delgado et al 199423 & 1972- 1990 & 28 \\
\hline 14. & Caron et al.1971 & 1930- 1966 & 43 \\
\hline 15. & Mark et al.1991 & $1955-1987$. & 18 \\
\hline 16. & Junior et al. 2003 & - & 24 \\
\hline 17. & Lewis et al.1997 & 6 years & 12 \\
\hline 18. & Clark et al1983 & 1916-1976 & 66 \\
\hline 19. & Forteza et al.1986 & 1967- 1985 & 9 \\
\hline 20. & Lindqvist et al 1986 & 1953-1983 & 9 \\
\hline 21. & Ajagbe et al 1986 & 1960- 1984 & 21 \\
\hline 22. & Bertoni et al. 1991 & $1950-1987$ & 28 \\
\hline 23. & Doval et al. 1997 & 1986-1992. & 8 \\
\hline 24. & Tanzawa et al. 1991 & 1930-1989 & 114 \\
\hline 25. & Slootweg and Muller1985 & 1950- 1983 & 18 \\
\hline 26. & Ryan et al 1986 & 1973- 1983 & 24 \\
\hline 27. & Daw et al.2000 & $\begin{array}{c}1962 \text { - January } \\
1998\end{array}$ & 28 \\
\hline 28. & Can Soc OLHNSOSG & - & 35 \\
\hline 29. & August et al1997 & $1967-1991$ & 30 \\
\hline 30. & Smith et al.2003 & 1985 to 1996. & 496 \\
\hline 31. & MardingerOet al 2001 & 1989 to 1998 & 14 \\
\hline 32. & Nissanka et al. 2007 & 1993 to 2003 & 19 \\
\hline 33. & Bennett et al. 2000 & $\begin{array}{c}30 \text { years from } 1968 \\
\text { to } 1998\end{array}$ & 25 \\
\hline 34. & McHugh et al.2006 & - & 21 \\
\hline 35. & Fernandes et al2007 & 1993 to $2003 .$. & 16 \\
\hline 36. & Jasnau et al.2008 & 1977 to 2004 & 49 \\
\hline 37. & Guadagnolo et al.2009 & $1960-2007$ & 119 \\
\hline 38. & Ogunlewe et al 2006 & $1983-2003$ & 17 \\
\hline 39. & Huber et al.2008 & $1974-1999$ & 14 \\
\hline 40. & Oliveira et al 1997 & - & 17 \\
\hline 41. & Smeele et al 1994 & 1969-1993 & 14 \\
\hline 42. & Wanebo et al 1992 & 1982- 1990 & 29 \\
\hline 43. & Garrington et al. 1967 & 1967 & 56 \\
\hline 44. & Ha et al.1999 & $1946-1998$ & 17 \\
\hline 45. & Thiele et al.2008 & $1990-2004$ & 12 \\
\hline 46. & Mark et al.,1991 & 1955-1987 & 18 \\
\hline 47. & Snehal G. Patel et al2002 & 1981 and 1998 & 44 \\
\hline 48. & Shyam R. Gadwal2000 & 1970 and 1997 & 22 \\
\hline 49. & Panizzoni et al 1992 & 1980- 1990 & 11 \\
\hline 50. & Takahama Junior et al 2003 & 1958- 2001 & 23 \\
\hline 51. & Padilla and Murrah2011 & - & 7 \\
\hline 52. & Huh et al.2011 & 1983 to 2008 & 12 \\
\hline 53. & $\begin{array}{c}\text { Chaudhary M, Chaudhary } \\
\text { SD.et al2012. }\end{array}$ & 1967 to 2010 & 300 \\
\hline 54. & Peter M Nthumba2012 & $\begin{array}{c}1992 \text { and May } \\
2011\end{array}$ & 235 \\
\hline 55. & PrabhuS,et al2013 & $1990-2010$ & 13 \\
\hline
\end{tabular}

Osteosarcoma may develop in pre-existing conditions. But the present case appears to have developed de novo as no history of pre-existing disease was associated which makes this report unusual. ${ }^{9,22,23}$ History of extraction of second molar, one year back, could be considered as predisposing factor as extraction wound 
didnot heal and the swelling appeared at the same site.Similar findings were reported by Prabhu. S .et al ${ }^{21}$ as well as Daffer ${ }^{24}$ in their studyin which many patients had a history of extraction before the onset of the lesion. ${ }^{2}$ Nissanka et al also showed history of previous dental treatments mostly dental extractions. The reason for this is most likely to be the rapid growth of tumour immediately after extraction, a phenomenon often showed by bone tumours. ${ }^{25}$

As this case occurred in female, possibility of hyperthyroidism could be considered. Although this aetiology was not thought butCamilo Jimenez et al reported many patients in their case series of 1234 patients who had hyperthyroidism ${ }^{26}$

Oxygen or nutrient gradient may have a role on cell differentiation of undifferentiated mesenchymal precursors to osteoblastic,chondroblasticorfibroblastic pathway. Furthermore factors such as angiogenesis or local biological mediators may have effects on localized area differentiation. ${ }^{27}$

The fibroblastic type is very rare, especially in the jaws. ${ }^{8,12}$

Variousother aetiologies have been mentioned in the literature such as genetic predisposition ${ }^{4}$, bone dysplasias $^{3}$, Li-Fraumeni Syndrome, Rothmund-Thomson syndrome ${ }^{4,6}$ fluorides $^{28}$, Radiotherapy ${ }^{29}$, cancer stem $^{1}$ cell hypothesis. ${ }^{30}$, hyperparathyroidism ${ }^{26}$, viral ${ }^{31}$, osteitisfibrocystica, Hereditary Retinoblastoma ${ }^{1}$ Trauma $^{2}$, chemical factors, which include beryllium compounds and methylcholanthrene, and Rapid bone growth.$^{32}$

Unique feature of the disease was changing radiographic pattern form mixed to completely radiolucent in between two surgeries whichcaused much diagnostic predicament as jaw osteosarcomas does not necessarily show classic radiographic signs such as Codman's triangle and Garrington'ssign as seen in osteosarcoma of the long bones. Since swelling showed mixed radiolucency and radio opacity before the first surgery, a provisional diagnosis of fibrous dysplasia was consideredalthough it is rare in mandible. Malignancy was not considered initially as there was no historyof underlying pre-existing disease. Lesion recurred extensivelyandsubsequently became completely radiolucent suggesting mesenchymal malignancy or fibrous dysplasia turning into fibroblastic variant of osteosarcoma. ${ }^{10,19}$ The differential diagnosis of fibroblastic osteosarcoma should include, Ewing's sarcoma, bone metastasis, fibrous dysplasia in early stage , osteomyelitis, and even lesions that do not usually affect the jaw bones, such as fibrosarcoma, leiomyosarcomas, or rhabdomyosarcomas. ${ }^{33}$

Misdiagnosed cases of osteosarcoma frequently reported in various literatures.Rosilene et al., reported a case of osteosarcoma of mandible initially resembling of periapical lesion and after initial endodontic treatment, a significant increase in the size of the lesion occurred ${ }^{34}$ PriyankaDebta ${ }^{35 \text { et }}$ al also misdiagnosed a case of osteosarcoma as benign cystic lesion which recurred aggressively to cause extensive destruction. CD Mounesh at el misdiagnosed asneurofibroma ${ }^{23}$.

It could be hypothesized that the first lesion presented by the patient was a low-grade central osteosarcoma, since this neoplasia is often misdiagnosed radiographically, clinically and microscopically as fibrous dysplasia. Furthermore, low-grade central osteosarcomas characterized by high incidence of local recurrence after inadequate surgical removal and can transform into higher-grade osteosarcoma. Another hypothesis is the malignant transformation of fibrous dysplasia, a rare but possible event. ${ }^{37}$ although fibrous dysplasiais comparatively rare in mandible. ${ }^{35}$

Recurrence after inadequate surgical removal is a frequent phenomenon as reported by $\operatorname{siew}^{38}$ and abbas ${ }^{3}$;ourcase also recurred within 2 months.

Shah et al, Bennet et al, Hauben et al and Cavalcanti found high numbers of craniofacial osteosarcomas with fibroblastic variant behaved more aggressively. ${ }^{39,40,41,42}$

As per literature various histochemical stains have been used to study thehistopathological features of osteosarcoma for e.g. picrosirius red, improved Ponceautrichrome and Masson trichrome staining .Similar findings were noted in our study using MassonsTrichrome and Van Giessen's Staining. ${ }^{43}$

The growth in this case was so rapid that in contrast to the literature, the time span between diagnosis and death was only few months, a feature uncommon for gnathic lesions ${ }^{1,25}$

\section{Conclusion}

It can be concluded that misdiagnosis is very common in osteosarcoma of the jaw as this case exhibited unusual features like changing radiographic patterns , de novo origin, recurrence, extraction as predisposing factor, a rare fibroblastic variant with scanty osteoid resulted in missing classical sign of osteosarcoma .Besides adding this case of osteosarcoma of the mandible in the dental literature, this article would catch the attention of the dental professionals so that they may approach such cases with greater concern and diagnose them at an early stage for better prognosis. 


\section{References}

[1]. HelenTrihia and Christos Valavanis (2012). Histopathology and Molecular Pathology of Bone and Extraskeletal Osteosarcomas, Osteosarcoma, Dr. Manish Agarwal (Ed.), ISBN: 978-953-51-0506-0, In Tech, Available from :http://www.intechopen.com /books/osteosarcoma/ histopathology-and-molecular- pathology-ofbone-and-extraskeletalosteosarcomas

[2]. Devi CharanShetty, PuneetAhuja, Aadithya B. Urs\&RupinderKaurHistopathological Variants of Jaw Osteosarcoma International Journal of Pathology; 2009; 7(2): 98-101985 www.jpathology.com/.

[3]. Mashhadi Abbas F. DDS. MS.,Moshref M. DDS. MS., Hooshmand B. DDS., Sargolzaei S. DDS. MS., Bagheri A. DDS. MS., Mashhadi A. DDS. MS., Rahimi H. Fibroblastic Osteosarcoma in mandible: A rare case report and review of literature journals.sbmu.ac.ir/djen/article/view/1276/1200

[4]. MohsenyAB . Bone: Conventional osteosarcoma. Atlas Genet CytogenetOncolHaematol. April 2008 .URL http://atlasgeneticsoncology.org/Tumors/convosteoid5344.html

[5]. World Health Organization classification of tumours. pathology and genetics of tumours of soft tissue and bone. Fletcher CDM, Unni KK, Mertens F, eds. Lyon: IARC Press; 2002p264-85.www.iarc.fr/en/publications/pdfs-online/pat-gen/bb5/BB5.pdf

[6]. Luiz Antonio Guimarães Cabral Imprint cytology of osteosarcoma of the jaw: a case report Journal of Medical Case Reports Journal of Medical Case Reports 2009, 3:9327 http://www.jmedicalcasereports.com/content/3/1/9327

[7]. Khorate MM, Goel S, Singh MP, Ahmed J (2010) Osteosarcoma of Mandible: A Case Report and Review of Literature. J Cancer SciTher 2:122-125. doi:10.4172/1948-5956.1000036

[8]. AnithaBojan, Winnifred Christy, SumathyChanmougananda, KannanAshokan. Osteosarcoma of mandible: a case report and review of literature. Journal Of Clinical And Diagnostic Research [Serial Online] 2012 May [Cited: 2013 Dec 30 ]; 6:753757ID: JCDR/2012/4207:0058

[9]. DengraS. ,Sharma N. , TekadeS. Fibroblastic Osteosarcoma in Mandible: A Rare Case Report and Review of Literature JPFA. Vol. 26. December. 2012,162-165

PII: S0970-2199(12)64003-X doi:10.1016/S0970-2199(12)64003-X

[10]. Norman Jaffe, Ajay Puri, and Hans Gelderblom, “Osteosarcoma: Evolution of Treatment Paradigms,"Sarcoma, vol. 2013, Article ID 203531, 7 pages, 2013

[11]. A Narwal, AHooda, R Sen, V Singh, A Gupta, S Bala, D Sethi. ParaostealOsteosarcoma of Mandible: A Case Report. The Internet Journal of Otorhinolaryngology. 2010 Volume 13 Number 1. http://ispub.com/IJORL/13/1/10197

[12]. Charles T Mehlmanosteosarcoma (Overview)EmedicineSurgery, 2011 http://emedicine.medscape.com/article/1256857-overview

[13]. Garrington GE, Scofield HH, Cornyn J, Hooker SP (1967) Osteosarcoma of the jaws. Analysis of 56 cases. Cancer 20: 377-391 PMID:5228026

[14]. Clark JL, Unni KK, Dahlin DC, Devine KD (1983) Osteosarcoma of the jaw.Cancer 51: 2311-2316 PubMed ID: 6573939

[15]. Bertoni F, Dallera P, Bacchini P, Marchetti C, Campobassi A. The Istituto Rizzoli- Beretta experience with osteosarcoma of the jaw. Cancer. 1991;68(7):1555-1563. PMID: 1893357

[16]. Batsakis J. Osteogenic and chondrogenic sarcoma of the jaws. Ann. Otol. Rhinol. Laryngol. 96:474-475,1987. PMID:3476008

[17]. H. van den Berg, , W. H. Schreuder, and J. de Lange Osteosarcoma: A Comparison of Jaw versus Nonjaw Localizations and Review of the Literature Sarcoma. 2013; doi: 10.1155/2013/316123

[18]. Ricardo J. Padilla and Valerie A. Murrah "The Spectrum of Gnathic Osteosarcoma: Caveats for the Clinician and the Pathologist". Head Neck Pathol. 2011 Mar;5(1):92-

[19]. Prabhu S, Jose M, Iyengar S. Gnathic Osteosarcoma: A Retrospective Analysis over 20 Year Period.KathmanduUniv Med J 2013;41(1):37-40

[20]. Chaudhary M, Chaudhary SD. Osteosarcoma of jaws. J Oral MaxillofacPathol 2012 6:233-8.DOI: 10.4103/0973-029X.99075

[21]. Sukumaran Anil, Anitha P. Krishnan, and R. Rajendran Osteosarcoma of the Mandible Masquerading as a Dental Abscess: Report of a Case Volume 2012 (2012) doi:10.1155/2012/635062

[22]. EsraYeşilova,a FarukAkgünlü Osteosarcoma: A Case Report Eur J Dent. 2007 January; 1(1): 60-63.PMID:19212500 PMCID:PMC2612954

[23]. Mounesh Kumar C D, Suresh K V, Pramod R C, Baad R, Kumar SP, Karthik B. Osteosarcoma of maxilla mimicking neurofibroma. Clin Cancer Investig J [serial online] 2012 [cited 2013 Jun 25];1:233-5.DOI: 10.4103/2278-0513.106274

[24]. Richard H. Daffner ,Karl R. FoxR. Kent GaleyFibroblastic osteosarcoma of the mandible Skeletal Radiol (2002) 31:107-111DOI 10.1007/s00256-001-0438-z

[25]. Nissanka EH, Amaratunge EAPD, Tilakaratne WM. Clinicopathological analysis of osteosarcoma of jaw bones. Oral Diseases. 2007;13(1):82-87. PMID:17241435

[26]. CamiloJimenez,Ying ET AL Primary Hyperparathyroidism and Osteosarcoma: Examination of a Large Cohort Identifies Three Cases of Fibroblastic Osteosarcoma Journal of Bone and Mineral Research Volume 20, Issue 9, pages 1562-1568, September 2005DOI: 10.1359/JBMR.050507

[27]. F. Baghaie and P. Motahhary osteosarcoma of the jaws: a retrospective study ActaMedicaIranica, Vol. 41 No. 2 (2003) : http://journals.tums.ac.ir/abs/1052

[28]. Yeung CA."A systematic review of the efficacy and safety of fluoridation". National Health and Medical Research Council (Australia) (2007). Evid Based Dent (2008). 9 (2): 39-43 doi:10.1038/sj.ebd.6400578 . PMID 18584000.

[29]. Sayin, B., Yildirim, N., Vural, M., Dede, D.. Osteosarcoma of the maxilla. Radiology and Oncology, North America, 39, jun. 2005. Available at: 〈http://ojs.szd.si/index.php/ro/article/view/1295>.

[30]. Valerie A Siclari and Ling Qin ${ }^{*}$ Targeting the osteosarcoma cancer stem cell Journal of Orthopaedic Surgery and Research 2010, 5:78 doi:10.1186/1749-799X-5-78

[31]. Dr. Peter Buecker, Dr. Mark Gebhardt, and Dr. Kristy Weber "Osteosarcoma "Electronic Sarcoma Update Newsletter(ESUN)February 2005 - Volume 2,

Number 1 V7N5 ESUN

[32]. PieroPicciOsteosarcoma (Osteogenic sarcoma) Orphanet Journal of Rare Diseases 2007, 2:6 ,1750-1172-2-6 doi:10.1186/1750$1172-2-6$

[33]. Osteosarcoma From Wikipedia, the free encyclopediahttp://en.wikipedia.org/wiki/Osteosarcoma.

[34]. Rosilene C. Soares1, Andréa F. Soares2, Lélia B.Souza3, Aldo L. V. dos Santos4, Leão P. Pinto5Osteosarcoma of mandible initially resembling lesion ofdentalperiapex: a case report Rev Bras Otorrinolaringol. V.71, n.2, 242-5, mar./apr. 2005 
[35]. PriyankaDebta, Fakir Mohan Debta, Jigna Shah, R.I. Dave and CharuGarg Multidisciplinary Approach for an Initially Misdiagnosed Case of Osteosarcoma in a edically Compromised PatientBritish Journal of Medicine \& Medical Research2(1): 94-104, 2012 www.sciencedomain.org/download.php?f=1328936013-Debtaetal.

[36]. Antony George, Varghese Mani, Sunil S, SreenivasanBs, Devi Gopakumar Osteosarcoma Of Maxilla: - A Case Of Missed Initial Diagnosis Oral \& Maxillofacial Pathology Journal [ Ompj ] Vol 1 No 1 Jan- Jun 2010

[37]. Márcio Bruno Amaral et al Ícaro Buchholz Advanced osteosarcoma of the maxilla: A case report, Med Oral Patol Oral Cir Bucal. 2008 Aug 1;13(8):E492-5.Article Number: 1111111533 @ Medicina Oral S. L. C.I.F. B 96689336 - ISSN 1698-6946

[38]. Siew-Tin Ong et al "Osteosarcoma presenting as an aggressive nodular mass in the region of the mandible"Journal of Oral Science, Vol. 46, No. 1, 55-59, 2004 PMID 15141725

[39]. Shah IC, Arlen M, Miller T. Osteogenic sarcoma developing after radiotherapy for retinoblastoma. Am Surg. 1974; 40: 485490.PubMed PMID: 5260185

[40]. Hauben EI, Weeden S, Pringle J, Van Marck EA, Hogendoorn PC. Does the histological subtype of high-grade central osteosarcoma influence the response to treatment with chemotherapy and does it affect overall survival? A study on 570 patients of two consecutive trials of the European Osteosarcoma Intergroup. Eur J Cancer. 2002; 38: 1218-1225 PMID:12044509

[41]. Bennett JH, Thomas G, Evans AW, Speight PM. Osteosarcoma of the jaws: a 30-year retrospective review. Oral Surgery, Oral Medicine, Oral Pathology, Oral Radiology, and Endodontics.2000;90(3):323-332. PMID: 10982954

[42]. Cavalcanti MGP, Ruprecht A, Yang J. Radiological findings in an unusual osteosarcoma in the maxilla. DentomaxillofacRadiol. 2000; 29:180-184. doi: 10.1038/sj.dmfr.4600519.

[43]. ZhonghuaZhong Liu ZaZhi. Application of histochemical staining in diagnosis of osteosarcomas]. 2005 Aug;27(8):48991.PMID:16188149 